\title{
An examination of effects of online scaffolding on students' online behavior and reaction
}

\begin{abstract}
Many researchers emphasize the effectiveness of online interaction with scaffolding in enhancing learning and performance. However, little is known about the students' behavior and reaction toward participation in online interaction, and whether scaffoldings have any effects on students' behavior or not. This study utilized times series data to investigate how students' usage of offered instructional tools in an online interaction could change their behavior as well as considered reaction. The results obtained by weekly observations confirmed that there were noticeable changes on students' behavior and reaction within scaffolding time with a same drop in both weeks 7 , and 10. Such changes seemed to be associated with differences in times they spent for using the instructional tools. Students' opinion about the usefulness of online scaffolding was consistent and aligned with their online behavior.
\end{abstract}

Keyword: Behavior; Learning performance; Online tutorial; Reaction 